\title{
Monistic and Dualistic Conceptions of the Stellar Universe*
}

\author{
By C. V. L. Charlier, Observatory of Lund, Lund, Sweden
}

1. The last ten years have brought about the gradual development of two diametrically opposed ideas in regard to our stellar universe-ideas which may be fitly charaoterized by the distinction implied in the title of this article. According to the monistic conception, the stellar universe is thought of as a single system, which, if not really in equilibrium, is well on the road to such a state. Others maintain that the stellar system can be better explained as a mixture of two systems, which are in the process of blending. the one with the other, and giving birth to a stellar universe which is far from being in what we should call in mechanics a stable state of distribution. It is asserted by both sides that they have established their opinions upon facts; and, in truth, the interpretation of the latter is so intricte that we are able, in many instances, (to use them) to support first one and the the other hypothesis.

For Herschel and the astronomers of the last cen tury the monistic point of view was the natural point of view. It was only when Kapteyn in 1904 set forth his celebrated theory of two star streams, that we seriously doubted the monistic conception of the stelar universe and demanded if it was adequate. I truth this hypothesis of Kapteyn seemed to explain in a simple way the anomalies in the proper motions of the stars to which Kobold some years before had directed the attention of astronomers. However, only three years after that, we find Schwartzschild showing that the same phenomena might be explained without giving up the monistic conception. He, nevertheless, was unable to prove that his hypothesis was superior to that of Kapteyn.

Since then each new discovery in stellar astronomyand there have been many recently, especially in the field of stellar spectroscopy-has been discussed from the point of view of one or the other of the two hypotheses.

It is justifiable first to say that the monistic conception has been recently revived and its foundation strengthened by the relation which has been established between the motions of stars and the kinetic theory of gases which has been developed by Clausius and Maxwell. This analogy was first suggested by $\mathrm{Sir}$ William Thomson (Lord Kelvin). But Poincaré (in his Hypotheses cosmogoniques, 1911) was the first to really try to apply the kinetic theory to the stars. H was not concerned there, however, with the fundamental problem in connection with the kinetic theoryin knowing the frequency of the distribution of stella velocitles. The problem has been approached more recently by Halm and Jeans and the writer, who has furthermore made great use of the kinetic theory in the lectures which he has given at Lund during the last two years.

The main idea in the kinetic theory is the following: Consider a group of stars made up of a great numbersome millions of single ones. The motion of one individual star is governed by the attraction of the others acconding to Newton's law. It is evidently impossible to consider exactly the attraction of each of the stars, partly because the work would be overwhelming on account of the calculation, and partly because we never know, and never will be able to know, with sufficient precision the relative position and the velocity of the stars. Therefore, we approach the problem in another way. The attraction of each individual star depends apparently, for the most part, upon the attraction of the whole of the group. The distances between the stars are great, and the orbit of an individual is, at least for a long time, the same as that of a particle moving in a field of force having at all points the same potential as the entire group. If the density of distribution of the stars and of the distribution of velocities at each point of the group remains unaltered, the system may be sald to be in dynamical equillibrium. If this equlibrium does not exist, the density of distribution varies from time to time.

Even in the case of very diffuse groups of stars we cannot be sure that the star under consideration will not pass from time to time, though perhaps at very long intervals, very near to another star so that its orbit will be suddenly and completely changed. Since the influence of these passages-in the kinetic theory of gases they are called collisions-cannot be analyzed by means of ordinary mechanics, we study it by the -Translated by Mise E. W. Wickham, from Bcientia 1917, theory, known also as "statistical mechanics." This theory shows that the passages in the course of time, smooth out the differences in the velocities of the stars, until at last an equilibrium-a statistical equilibriummay be reached. The velocities in each part of the group are then distributed according to Maxwell's law. Another important question is that connected with the mixture of stars of different masses. Here an equipartition of energy is produced, such that the product of the mass of a star by the square of its velocity is, on the average, the same for stars of all sizes. Thus, in the long run, the most massive stars have the smallest, while the smallest ones acquire the greatest mean velocities.

Let us pass now from the kinetic theory to the comparison of the monistic and dualistic theories of the universe. In the following table I have collected the principal problems which the two theories have tried to explain and have added a short description of the solution:

$$
\begin{aligned}
& \begin{array}{l}
\text { Problem. } \\
\text { state of mot }
\end{array} \\
& \begin{array}{l}
\text { Monistic theory. } \\
\text { The universe is }
\end{array} \\
& \text { almost in } d y \text {. } \\
& \text { namical equilib- } \\
& \text { rium and is con- } \\
& \text { tistical equllib. } \\
& \text { Dualistic Theory. } \\
& \text { nor statistical equl- } \\
& \begin{array}{l}
\text { nor sta } \\
\text { llbrium. }
\end{array} \\
& \begin{array}{l}
\text { Apeed and Bpeo- The mean speed } \\
\text { tral type. } \\
\text { depends up o o } \\
\text { the maseed of ith the age of }
\end{array} \\
& \text { the maseres of } \\
& \text { constant). } \\
& \begin{array}{l}
\text { Develop } \\
\text { a star. }
\end{array} \\
& \begin{array}{l}
\text { (meteoric dust) } \\
\text { red, ge } 110 \text { o } \\
\text { red, stars (M. } \\
\text { white gellow, } \\
\text { K. G. F. A. B. }
\end{array} \\
& \begin{array}{l}
\text { K. G. F. A. B. } \\
\text { A. F. G. K. M.) }
\end{array} \\
& \begin{array}{l}
\text { yellow, red stars } \\
\text { (B. A. F. G. K. }
\end{array}
\end{aligned}
$$

The discussion of the five problems of the preceding table would be too long for an anticle in this journal, so I shall limit myself to the first one. In a general way I will give all the arguments in favor of the kinetic theory, and discuss the objections which hav been made to its application to the stellar universe. The most important of these have been made by Jeans, Eddington and Poincaré, and I will discuss them here in their order.

2. Jean's Objection: The stellar universe may be in statistical equilibrium, and, in that case, also in dynam cal equilibrium; or it may be only in dynamica equilibrium, or, according to the dualistic theory, it need be in neither dynamical nor statistical equilibrium. It is evident from the proper motions of the stars that it is not in statistical equilibrium. We shall see later, nevertheless, that it is manfestly approaching that state. In regard to the two other possibilities, the question was studied in 1915 by Jeans. The result of his researches was that "star streams, such as we have observed in the universe, do not seem to be in accord with the supposition, that our universe is in a stable state." Accepting the dualistic theory, I examined the problem at the same time as Jeans, in my lectures in 1915, and arrived at the opposite conclusion. To be more precise, I obtained the same mathematical (and mechanical) result as Jeans, but we differ in regard to the application to the astronomical problem. A far as I can see, this is the explanation. For a stable state it is necessary-as a mathematical analysis shows-for the ellipsoid of velocity to be a spheroid whose axis of revolution is perpendicular to the radius vector drawn from the center of the universe. The observations show in fact that the surface of velocity is approxfmately an ellipsoid of revolution. What is the angle between the axis or revolution, and the radius vector from the center? We may have different opinions upon this point, in consequence of the diffculty which exists in determining the position of th center. Mr. Jeans thinks that this axis is directe towards the center (agreefng with Turner) while according to my opinion it is approximately (but no exactly) perpendicular to the radius vector as the theory demands. Since my determination of the cener of the universe, from the distribution of helium stars (June 1916) was not known to Mr. Jeans at the date of the publication of his article (December 1915), I hope that he will find some reasons to modify his conclusions upon this point.

3. Let us now see to what extent we can accept the hypothesis that the universe is really on the road to a state of statistical equilibrium. This question has also been dealt with by Jeans, who, as the author of $L a$ theorie dynamique des gaz-the best treatise on the kinetic theory known to the author-is a peculiarly competent authority upon the subject. In order to measure the velocity with which a disturbed gas reaches a stable state, Maxwell made use of a quantity which he called the "relaxation time." The greater this time, the slower is the restoration to the stable state by the collisions of particles. It should be noted that the time necessary to establish (approximately) a statistical equilibrium is always greater than the "relaxation time," but one may consider the two times as being of the same order of magnitude. For gases, the time is of the order of $10^{-10}$ seconds. For stars, Jeans found the enormous value of $10^{14}$ years. Applying Newton's law to the problem (instead of Maxwell's law of repulsion, which Jeans used) I found the value $10^{16}$ years for the same quality-a value of the same order of magnitude as Jeans. In other words, this shows that it would take the stellar universe some billions of years to arrive at statistical equllibrium by the collisions of stars. Jeans thinks this time too long "even for astronomy," and concludes that "there can "even for astronomy," and concludes that "there can at a final stable state like that which the theory of gases has made us acquainted." Without doubt the period is very long and exceeds all our previous ideas as to the age of the stars. But have these ideas a foundation of sufficient accuracy? Have we taken scrupulous account of the phenomena of radiation? I think not. In any case, it may be wise to examine the facts showing the influence of these collisions of stars upon the distribution of their velocities.

4. And indeed, facts of this kind are not lacking. In the following table, from Gyllenberg, are given the semi-axes of the ellipsoids of velocity.

SEMI-AXES OF THE ELLIPSOIDS OF VELOCITY OF STARS OF

\begin{tabular}{lccccc}
\multicolumn{7}{c}{ DIFFERENT } & SPECTRAL TYPE. \\
Color & Type & $a_{1}$ & $a_{2}$ & $a_{2}$ & \multicolumn{1}{c}{} \\
White stars & B \& A & 1.73 & 3.10 & 2.73 & 4.48 \\
Yellow " & F \& G & 3.02 & 4.95 & 3.67 & 6.86 \\
Red " & K \& M & 4.00 & 4.51 & 4.27 & 7.39
\end{tabular}

The figures represent siriometers per stellar year, the siriometer being equal to $10^{\circ}$ astronomical units, and the stellar year being equal to $10^{\circ}$ years. The axis $a$ is (approximately) directed to the center of the Milky Way; the axis $a_{2}$ is perpendicular to it in the Milky Way (toward the Vertex), and the axis $a_{z}$ is directed toward the pole of the Milky Way. Various researches made by Ludendorff, Shapley and others point to the conclusion that it is probable that the white stars have the greatest mass; next come the yellow, and the red ones have the smallest mass. Considering all these points we find:

1. The mean velocities $(. \delta)$ of the stars are propor tionally greater as the masses of the stars are small, which agrees with the law of equipartition of energy in the kinetic theory.

2. The largest axis $a_{3}$ is approximately perpendicular to the radius vector drawn from the center.

3. The other axes ( $a_{1}$ and $a_{3}$ ) are, at least for the yellow and red stars, almost of equal length, so that the ellipsoid of velocity is a spherold, located (almost) in the position necessary for dynamical equilibrium. 4. For the smallest stars (red) the three axes of the ellipsoid of velocity are almost of the same length, showing that these stars are already, at least approximately, in statistical equllibrium.

This seems to show that the "relaxation time" in a group of stars is proportional to the mass of the latter. These considerations are, indeed, purely provisional, for a separation of spectral types here taken together (B \& A, F \& G, etc.), is necessary before one can undertake a conclusive discussion. (Thus the ellipsoids of velocity of B type stars differ essentially from those of A type stars.) In any case, the preceding considerations seem to indicate that the "relaxation time," long as it may be, has already left some recognizable traces in the history of our stellar universe.

(To be concluded) 